\title{
EEG Based Headband for Emotion Detection
}

\section{Spoorthy S}

\begin{abstract}
EEG is the term used for recording the brain electrical activity. In Electroencephalography, the encephalon means brain. EEG measures electrical activity generated by thousands of neurons that exists in human brain. The brain electrical activity is measured in voltages. This paper is focused on recognizing emotion from human activity, measured by EEG signals. Making the computer more empathic to the user is one of the aspects of affective computing. With EEG-based emotion detection, the computer can actually take a look inside user's head to observe their mental state. A low power, low noise and high sensitive analog signal from brain decoded into filtered digital output. The decoder picks a low amplitude and a microvolt signal from brain and decodes it into a filtered and amplified output. As of thelatestattentiongiving fromexaminationteam in creatingsensitivecommunicationamong human beings and peripheral device, the proof of identity of emotive state of the previousdeveloped a necessity. Electro-encephalography establishedimportantconsideration from scientists, becausethey establish modest, inexpensive, transportable, and easily solving theidentification of mind states in this paper. [2] In this paper, it provide a comprehensive overviewfrompresent works in emotion detection using EEG signals.
\end{abstract}

IndexTerms: Emotions,

Electroencephalography, Identification, Recognition.

\section{INTRODUCTION}

Electroencephalography (EEG) is one effective way ofrecording andexaminingthebrainactivityduetoitsease of use,affordable cost and fine resolution. Due to brain activity neurons get fired producing higher electrical potential which is recorded by the electrodes attached to the scalp of a human being. The measuresdiffer due tovarying levelsof cognitive stimuli. EEG is advantageous due tohigh temporal resolution which helps to record differencesin cognitive activity based on millisecond gauge. Hence, EEG measurements are insight of the cognitive situations of the participant. However, EEG is sensitive to noise from electrical equipment, typicallyworkingin controlled lab environments and not so effectivein realworldsituations.

Human-computer interferenceislearningof communication among people and computers. HCI aims to produce a plan that produces a good suitable among the operator, the machine and the necessary services in order to attaina certain performance in relationsof quality and optimality of the facilities.

Revised Manuscript Received on December 30, 2019.

* Correspondence Author

Spoorthy S, Department of Electronics and Communication Engineering, NIE Institute of Technology, Mysuru, India.

Email: spoorthy1997@gmail.com

(C) The Authors. Published by Blue Eyes Intelligence Engineering and Sciences Publication (BEIESP). This is an open access article under the CC BY-NC-ND license (http://creativecommons.org/licenses/by-nc-nd/4.0/)
HCI is the highestactive examinationfieldin computer science presently. Modern types of human-driven and humancentric communication with digital media have opened new possibilities to modernize the different areas of human life such as learning, working etc. Human-computer interface applications have improved the significance of emotion recognition as emotions are vital in daily life ofhumans.

Electroencephalography is a signal illustration of brain actions. The signal waves keeps the valuable evidence about the current situations of brain. This is the non-invasive method for brain imaging which offers electrical potential footage for the surface of the scalp because of the electrical movement of the large groups of neurons in the brain. Non-invasive is a technique where the human body is not entered or cut open as during surgical operations or therapeutic surgery. Invasive method is opposite to non-invasive.

EEG evaluates brainwaves of varying frequencies within the brain. In specific location electrodes are situated on the scalp to sense and record the electrical compulsions within the brain. The recordings are the sum of volume conductor fields formed by millions of interconnecting neurons. Depending on the positions of the placed electrodes, the EEG signal varies.

\section{OPERATIONAL PRINCIPLE}

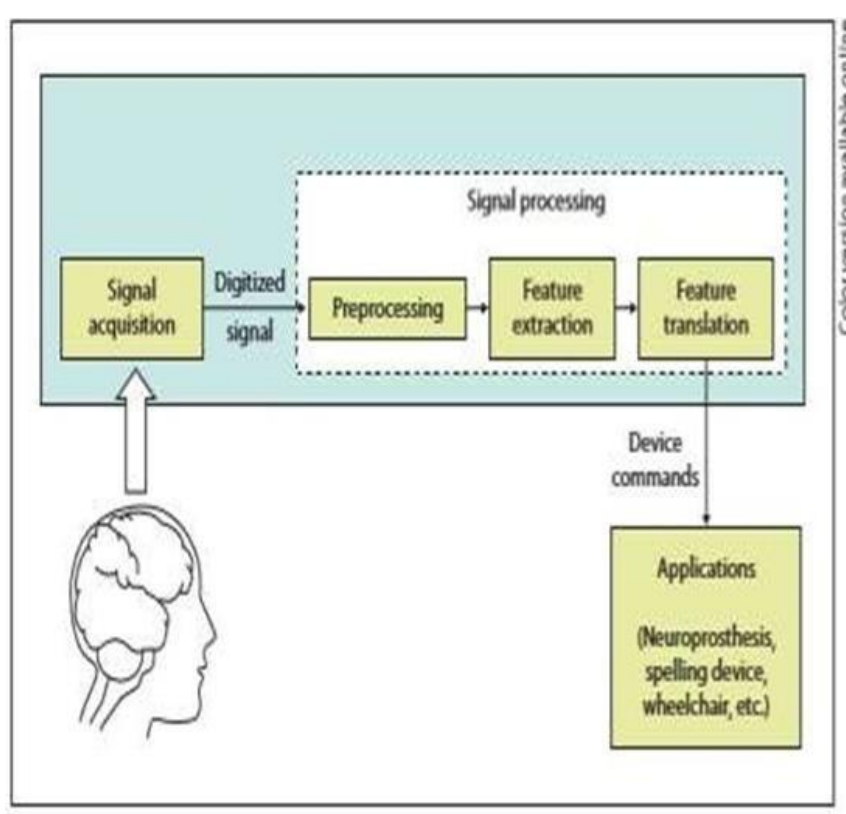

Fig. 1. Overview of EEG System

Published By: 


\section{EEG Based Headband for Emotion Detection}

Figure.1 includes a collection of recognizing and signalprocessing apparatuses that allows the acquisition and examination of brain actions in order to create a dependable, straight communication channel in the middle of the mind and an peripheralcomponentlike computer or a neuroprosthesis system, etc.[13] The block diagram involves of 3 main parts: signal acquisition, signal processing and applications. By electrodes situated on the scalp, the brain signals are noted (noninvasive BCI systems). A signalextracted by these electrodes are generally covered by sounds and differentobjectscontainingcontrol linesinterfering, electrode dis-placement, topicactivities, electrode or wire connection, and physical interference from visual, muscular, respiratory, and cardiac events. To remove these unwantedsounds and artifacts is a compulsory preprocessing step. The feature extraction and translation components are to translate the attained brain signal into measureableindication of some neurological effects of interest. The informative features are converted into controllerpulseslikegraphicresponse to the operator, a continuouselectricalsignals to regulatorperipheralcomponents.[13]

Figure.2 shows how to recognize emotions using EEG signals we need to achieve the subsequent steps:

- $\quad$ The operator must be visible to the stimulus beingverified.

- The voltage fluctuations observed in the brain of the operator are noted.

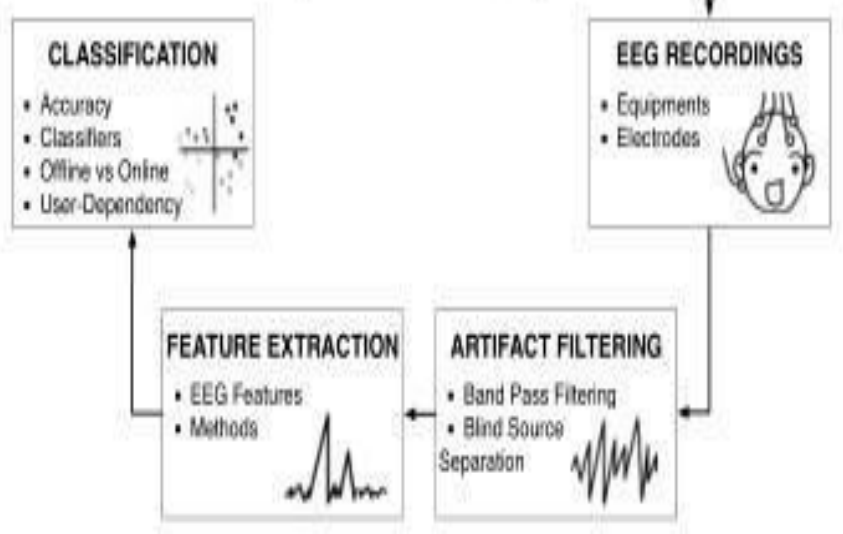

Fig. 2. Process of emotion recognition using EEG

- Asound and artifacts from the recorded signals areremoved.

- The resultant data is evaluated and the applicable features are extracted.

- A distinguisher is skilledcreatedfrom training collection and fromcalculatedstructures, giving the evaluation of the human brain pulses extracted from origin.[2]

\section{METHODOLOGY}

A headband with three electrodes is allowed to be placed

On subject's head which recordthe variations on brain wave.

The electrode used is of dry silver chloride (AgCl)electrode.

From the electrode the obtained signal is filtered by band

pass filter and notchfilter. The filtered signal is then digitalized.

To recognize emotions using EEG signals we need to perform the

following steps:

- The user must be exposed to the stimulus being tested.

- The voltage changes observed in the brain of the user are recorded.

- The noise and artifacts from the recorded signals are removed.

- The resulting data is analyzed and the relevant features are

extracted.

\section{RESULTS}

\section{A. Analysis For EEGSignal}

The EEG signal can be classified into six significant Frequency waves as follows:

\section{Delta Waves}

Delta waves are the frequencies rangingtill 4 Hertz. It is observed to bemaximum in amplitude and the slowest waves. It can be observed in older people in slow wave sleep. Thishappensmainlyfrom subcortical lesions and also fromscatteringfrom diffuse lesions, deep midline lesions.

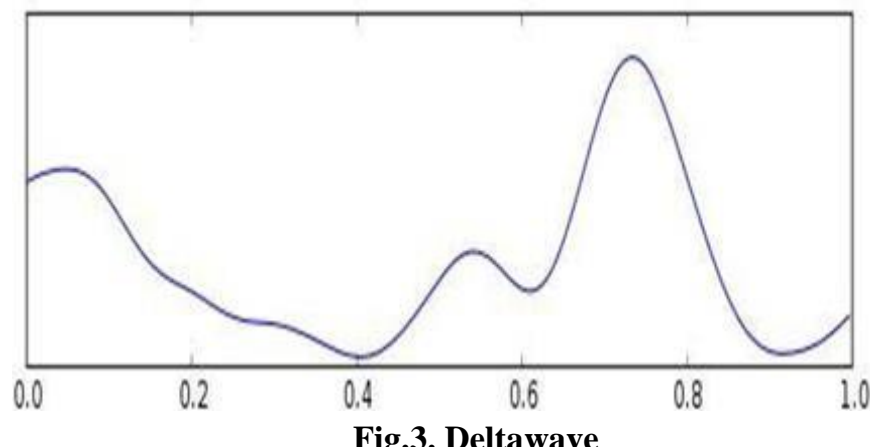

\section{Thetawaves}

Theta waves are the frequencies ranging from 4 Hertz to 7 Hertz. Theta are not observedgenerally in adult but seen in younger generations. This isobserved in tiredness, stimulation in grown-upkids and adults; this may have beenobserved from meditated brain. Additionalabnormal activity is seen from theta.[4] 


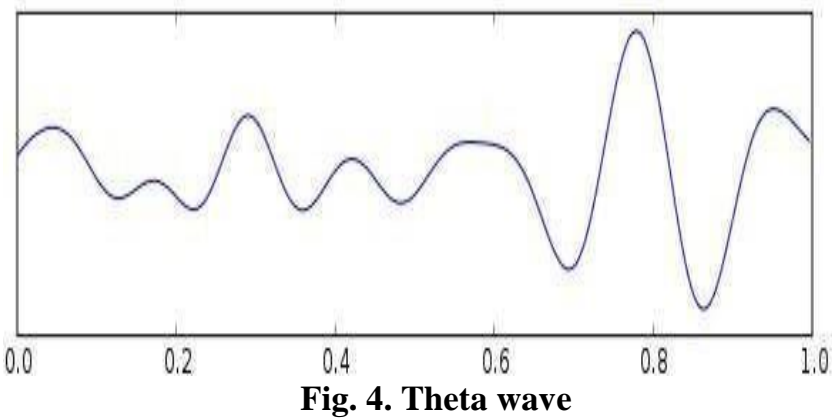

3. AlphaWave

Alpha waves are the frequencies varying from 8 Hertz to 12 Hertz. Hans Berger termed the main recurring EEG act he have seen this as the "alpha wave". These are the "posterior basic rhythm", observed from later areas from skull on either parts, advanced in amplitude on the leading region.[4]

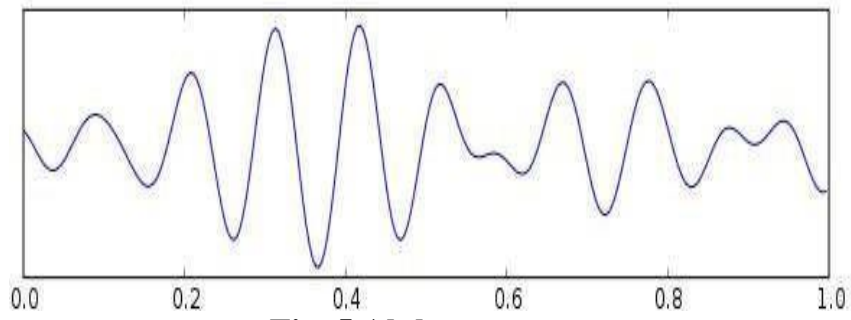

Fig. 5.Alpha wave

\section{BetaWave}

Beta waves are the frequencies varying from 12 Hertz to around 30 Hertz. It is observed on the either sides in symmetrical distribution and most obviously in frontal. Motor behavior are closely linked to Beta actions and are usually reduced during active movements. In the areas of cortical damage these are absent or reduced.

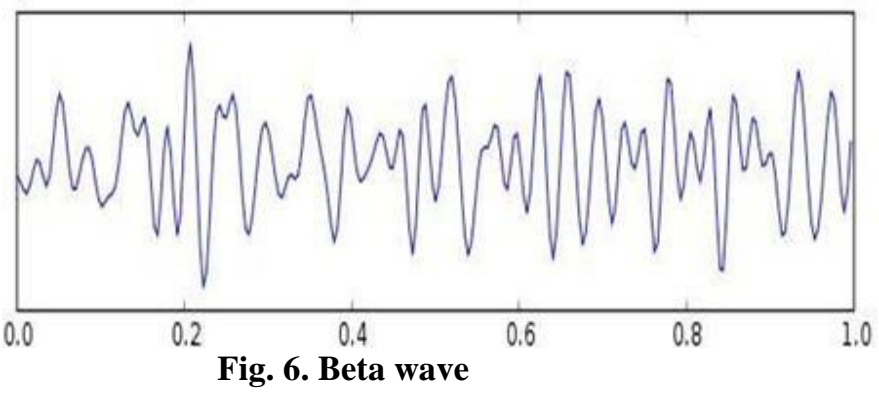

5. GammaWave

Gamma waves are the frequencies ranging approximately from 30

Hertz to 100 Hertz. Gamma waves are believed to signify collecting

Of dissimilar numbers of neurons collected to the system to determine the performance of a engine role.

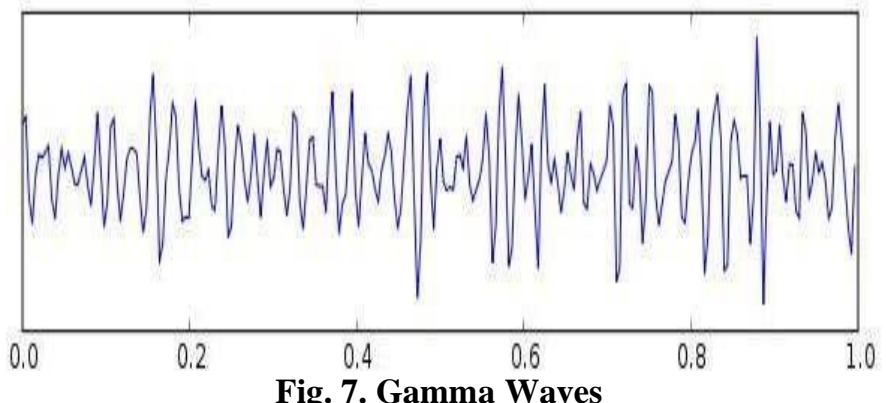

Fig. 7. Gamma Waves

6. Mu Wave

$\mathrm{Mu}$ waves from 8-13 Hertz and partially overlaps with supplementary frequencies. Relaxation state is the reflection of continuous shooting of motor neurons. Mu suppression is believed to imitate motor mirror neuron structures, because when an act is witnessed, the pattern extinguishes, probably because of the usual neuronal network and the mirror neurons network are not synchronized then they interfere with one another.

\section{B. WaveformObservation}

Laptop with a software which allows us to observe the analog waveforms.

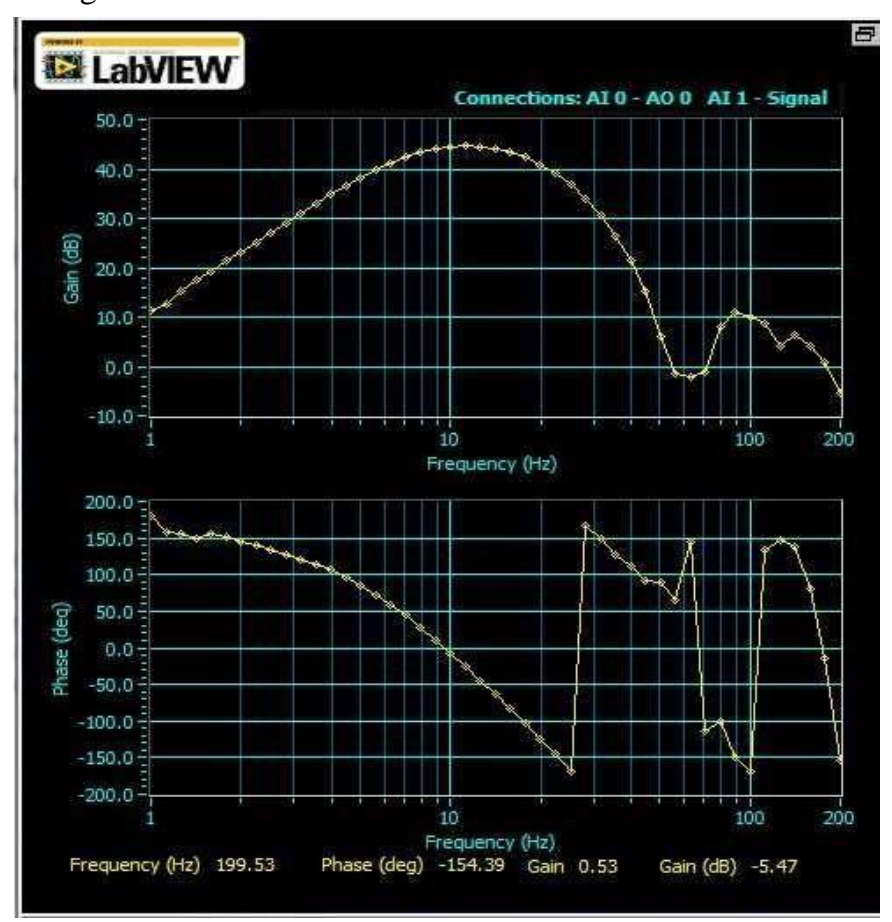

Fig.8. Expected Waveform

\section{CONCLUSION}

This paper object was to get the low power, low noise brain signal that can be used to control real world application. In this paper we learned about the technique to design and implement the low power low noise and high sensitive EEG portable system.

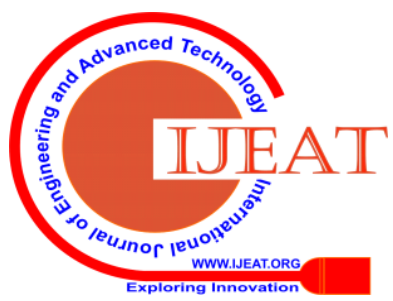




\section{EEG Based Headband for Emotion Detection}

This paper holds importance in almost every field of training especially in the field of medical and communication and control. We considered about the designing and behaviors of different electronic components in detail. That is the most important part of our filed. We considered wired and wireless protocol which is an interesting technology used in electronic market today. Finally, we have a Low power, Low noise and high sensitive portable brain wave decoding system that can be used for any real world applications.

\section{ACKNOWLEDGEMENT}

I would like to take this opportunity to express my utmost appreciation and sincere gratitude to our internal guideManjula A V, Asst. Professor, Dept of ECE, NIEIT, Mysuru-

18. Apart from that, I also wish to extend my warmest thanks to Dr. Archana N V, Professor and HOD, ECE Dept. NIEIT, Mysuru-18 and also VTU University for guiding and supporting me throughout my journey.

\section{REFERENCES}

1. "Identfying Ketamine Responses in Treatment-Resistant Depression Using a Wearable Forehead EEG"- Zehong Cao, Member, IEEE, ChinTeng Lin, Fellow, IEEE, Weiping Ding, Member, IEEE, Mu- Hong Chen,Cheng-Ta Li, Tung-PingSu.

2. Soraia M. Alarcao, Manuel J. Fonseca. "Emotions Recognition Using EEG Signals: A Survey", IEEETransactions on Affective Computing, 2019

3. "Emotion recognition based on high-resolution EEG recordings and reconstructed brain sources"- Hanna Becker, Julien Fleureau, Philippe Guillotel, FabriceWendling, Is-abelleMerlet, Laurent Albera Senior Member, IEEE.

4. Che-Wen Chen, Chia-Yi Chou, Jhing-Fa Wang. "The personal characteristics of happiness: An EEG study",2015 International Conference on Orange Technologies (ICOT), 2015.

5. Melnik A, Legkov P, Izdebski1 K, Kärcher SM, Hairston WD, Ferris DP, König P. Systems, Subjects, Sessions: To What Extent Do These Factors Influence EEG Data? Frontiers in Human Neuroscience. 30 March 2017. https://doi.org/10.3389/fnhum.2017.00150

6. De Vos M, Kroesen M, Emkes R, Debener S. P300 speller BCI with a mobile EEG system: comparison to a traditional amplifier. Journal of Neural Engineering, 11(3):1-8,2014.

7. John MS, Dimitrijevic A, PictonTW. MASTER: a Windows program for recording multiple auditory steady state responses. Computer Methods and Programs in Biomedicine. 61, 125-150,2000

8. Ducharme M. Développementd'uneplateforme de recherche portable pour électroencéphalographie intra- etcircum-auriculaire. Master's thesis (publication pending). École de technologiesupérieure - Université du Québec,2018.

9. Cone-Wesson B, Dowell RC, Tomlin D, Rance G, Ming WJ. The auditory steady-state response: Comparisons with the auditory brainstem response. J Am AcadAudiol. 13:173-83,2002.

10. PictonTW, John MS, Dimitrijevic A, Purcell D. Human auditory steady state responses. Int J Audiol. 42:177-219,2003.

11. Galambos R, Makeig A, Talmachoff PJ. A 40-Hz auditory potential recorded from the human scalp. Proceedings of the National Academy of Sciences of the United States of America. 78(4):2643-47,1981.

12. Validation and Benchmarking of a Wearable EEG Acquisition Platform for Real-World Application Olivier Valentin*, MikaëlDucharme, Gabrielle Crétot-Richert, HamiMonsarrat- Chanon, GuilhemViallet, AidinDelnavaz and JérémieVoix.

13. Chin-Teng Lin, Li-Wei Ko, Meng-Hsiu Chang, Jeng-RenDuann, JingYing Chen, Tung-Ping Su, Tzyy-Ping Jung."Review of Wireless and Wearable Electroencephalogram Systems and Brain-Computer Interfaces - A Mini-Review",Gerontology, 2010 Crossrefexport.arxiv.

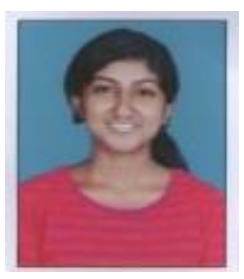

\section{AUTHORS PROFILE}

Spoorthy S, Department of Electronics and Communication Engineering, NIE Institute of Technology, Mysuru, India. Email: spoorthy1997@gmail.com 